\title{
A GENERALISED UNIQUENESS THEOREM AND THE GRADED IDEAL STRUCTURE OF STEINBERG ALGEBRAS
}

\author{
LISA ORLOFF CLARK, RUY EXEL, AND ENRIQUE PARDO
}

\begin{abstract}
Given an ample, Hausdorff groupoid $\mathcal{G}$, and a unital commutative ring $R$, we consider the Steinberg algebra $A_{R}(\mathcal{G})$. First we prove a uniqueness theorem for this algebra and then, when $\mathcal{G}$ is graded by a cocycle, we study graded ideals in $A_{R}(\mathcal{G})$. Applications are given for two classes of ample groupoids, namely those coming from actions of groups on graphs, and also to groupoids defined in terms of Boolean dynamical systems.
\end{abstract}

\section{INTRODUCTION}

Steinberg algebras were independently introduced in [22] and [10] and are closely related to the machinery established in [14]. This broad class of algebras provides a general model for Leavitt path algebras associated to directed graphs, Kumjian Pask algebras associated to higher-rank graphs, and discrete inverse semigroup algebras.

In this note, we use an analysis of the 'interior of the isotropy bundle' to prove a generalised uniqueness theorem. We also characterize the graded ideals for Steinberg algebras over groupoids equipped with a cocycle taking values in a discrete group. Applications are then provided in two broad classes of examples. Our presentation is as follows:

After establishing our notation and discussing some preliminary results in Section 2, we move to Section 3 where we prove a generalised uniqueness theorem for Steinberg algebras modeled after the $C^{*}$-algebra [6, Theorem 3.1(c)]. Our uniqueness theorem says that a Steinberg algebra homomorphism is injective if and only if it is injective on the interior of the isotropy group bundle. This generalises theorems [18, Theorem 5.2] and [11, Theorem 5.4] for Leavitt path algebras and Kumjian-Pask algebras respectively.

In Section 5, we characterize the graded ideals of a Steinberg algebra built from 'graded groupoids'. These are groupoids that come equipped with a homomorphism (or 'cocycle') $c$ into a discrete group such that the inverse image of the identity doesn't have too much isotropy. In this setting, we show that the graded ideals are precisely those generated by open invariant subsets of the unit space.

In the last two sections, we give two more classes of examples. Both are classes whose $C^{*}$-algebras have been constructed using the machinery of [14]. In Section 6 we consider groups acting on graphs, as defined in [17]; we describe the Steinberg algebra associated associated to such an action. This broad class of algebras includes the class of Leavitt path

2010 Mathematics Subject Classification. 16S99, 16S10, 22A22, 46L05, 46L55.

Key words and phrases. Groupoid $C^{*}$-algebra, Steinberg algebra, Graded ideal, Self-similar graph algebra, Boolean dynamical system.

The first named author was partially supported by Marsden grant 15-UOO-071 from the Royal Society of New Zealand. The second-named author was partially supported by CNPq. The third-named author was partially supported by PAI III grant FQM-298 of the Junta de Andalucía, and by the DGI-MINECO and European Regional Development Fund, jointly, through grant MTM2014-53644-P. 
algebras. We also get an algebraic analogue of Katsura's algebras $\mathcal{O}_{A, B}$. Finally, in Section 7 we construct a Steinberg algebra from a Boolean dynamical system, as introduced in $[7]$.

\section{Preliminaries}

Recall that a topological ${ }^{1}$ space $X$ is said to be zero-dimensional if the topology of $X$ admits a basis consisting of clopen (closed and open) sets. If, in addition, $X$ is locally compact, it is easy to see that $X$ also admits a basis formed by compact open sets.

In this work, we assume most topological spaces are locally compact, zero-dimensional and Hausdorff. If $X$ is such a space, and if $R$ is a unital commutative ring, we denote by

$$
C_{c}(X, R)
$$

the $R$-module ${ }^{2}$ formed by all locally constant, compactly supported, $R$-valued functions on $X$. Notice that an $R$-valued function is locally constant if and only if it is continuous once we equip $R$ with the discrete topology.

If $D$ is a compact open subset of $X$, the characteristic function of $D$, here denoted by $1_{D}$, is clearly an element of $C_{c}(X, R)$. In fact, every $f$ in $C_{c}(X, R)$ may be written as

$$
f=\sum_{i=1}^{n} a_{i} 1_{D_{i}}
$$

where the $D_{i}$ are compact open, pairwise disjoint subsets of $X$. The support of $f$, defined by

$$
\operatorname{supp}(f)=\{x \in X: f(x) \neq 0\}
$$

(we do not use closure in the definition of the support), is clearly a compact open subset.

If $X$ is as above, and $U \subseteq X$ is an open subset, then $U$ is also a locally compact, zero-dimensional, Hausdorff space. Moreover we may view $C_{c}(U, R)$ as a submodule of $C_{c}(X, R)$ as follows: given any $f$ in $C_{c}(U, R)$, let $\tilde{f}$ be the extension of $f$ to $X$ defined to be zero on $X \backslash U$. It is then easy to see that $\tilde{f}$ is continuous on $X$ and, in fact, that it lies in $C_{c}(X, R)$. The correspondence

$$
f \in C_{c}(U, R) \mapsto \tilde{f} \in C_{c}(X, R),
$$

is then an $R$-module isomorphism from $C_{c}(U, R)$ onto the submodule of $C_{c}(X, R)$ formed by the functions vanishing on $X \backslash U$. We may therefore view $C_{c}(U, R)$ as a submodule of $C_{c}(X, R)$.

Given a groupoid $\mathcal{G}$, we will always denote its unit space by $\mathcal{G}^{(0)}$, the set of composable pairs by $\mathcal{G}^{(2)}$, and its source and range maps by $s$ and $r$, respectively. A bissection in $\mathcal{G}$ is a subset $U \subseteq \mathcal{G}$ such that the restrictions of $r$ and $s$ to $U$ are both injective.

A Hausdorff topological groupoid $\mathcal{G}$ is said to be étale if $\mathcal{G}^{(0)}$ is locally compact and Hausdorff in the relative topology, and its range map is a local homeomorphism from $\mathcal{G}$ to $\mathcal{G}^{(0)}$ (the source map will consequently share that property). It is easy to see that the topology of an étale groupoid admits a basis formed by open bissections. In an étale

\footnotetext{
${ }^{1}$ Unless otherwise mentioned, all topological spaces (including topological groupoids) in this work are assumed to be Hausdorff.

${ }^{2}$ Even though $C_{c}(X, R)$ is also an $R$-algebra, relative to pointwise product, we will not always view it as such.
} 
groupoid one has that $\mathcal{G}^{(0)}$ is open in $\mathcal{G}$. If, in addition, $\mathcal{G}$ is Hausdorff, then $\mathcal{G}^{(0)}$ is also closed in $\mathcal{G}$. Throughout this paper all groupoids will be assumed Hausdorff.

Definition 2.2. An étale, Hausdorff groupoid $\mathcal{G}$ is said to be ample if $\mathcal{G}^{(0)}$ is zero-dimensional.

It is easy to see that the topology of an ample groupoid admits a basis formed by compact open bissections. En passant we deduce that an ample groupoid is also locally compact and zero-dimensional.

Definition 2.3 ([22], [10]). Given an ample, Hausdorff ${ }^{3}$ groupoid $\mathcal{G}$, and a unital commutative ring $R$, the Steinberg algebra associated to $\mathcal{G}$, denoted $A_{R}(\mathcal{G})$, is defined to be the $R$-algebra obtained by equipping $C_{c}(\mathcal{G}, R)$ with the convolution product

$$
(f g)(\gamma)=\sum_{\substack{\left(\gamma_{1}, \gamma_{2}\right) \in \mathcal{G}^{(2)} \\ \gamma_{1} \gamma_{2}=\gamma}} f\left(\gamma_{1}\right) g\left(\gamma_{2}\right)
$$

Since every element of $A_{R}(\mathcal{G})$ is a linear combination of characteristic functions of bissections, it is interesting to notice that

$$
1_{B} 1_{D}=1_{B D}
$$

whenever $B$ and $D$ are compact open bissections in $\mathcal{G}$.

If $\mathcal{G}$ is an ample, Hausdorff groupoid, and if $\mathcal{H}$ is an open subgroupoid of $\mathcal{G}$, then $\mathcal{H}$ is also ample and the natural inclusion

$$
A_{R}(\mathcal{H}) \rightarrow A_{R}(\mathcal{G})
$$

given by $(2.1)$, identifies $A_{R}(\mathcal{H})$ with a subalgebra of $A_{R}(\mathcal{G})$.

An elementary example of an open subgroupoid of $\mathcal{G}$ is $\mathcal{G}^{(0)}$, so we have that

$$
A_{R}\left(\mathcal{G}^{(0)}\right) \subseteq A_{R}(\mathcal{G})
$$

Due to the very special nature of the groupoid $\mathcal{G}^{(0)}$, the convolution product on $A_{R}\left(\mathcal{G}^{(0)}\right)$ coincides with the pointwise product.

Recall that an algebra $B$ is said to be $s$-unital when for every $r_{1}, r_{2}, \ldots, r_{n} \in R$, we can find $s \in R$ such that

$$
s r_{i}=r_{i}=r_{i} s, \quad \forall i=1, \ldots, n .
$$

Moreover, if $E$ is a given set of idempotent elements in $B$ such that the above $s$ can be taken in $E$, we will say that $E$ is a set of local units for $B$.

Lemma 2.6. Let $\mathcal{G}$ be a ample, Hausdorff groupoid. Then the family of all idempotent elements of $A_{R}\left(\mathcal{G}^{(0)}\right)$ is a set of local units for $A_{R}(\mathcal{G})$. In particular the latter is an s-unital algebra.

Proof. Given a compact open bissection $D \subseteq \mathcal{G}$, and given any compact open subset $C \subseteq \mathcal{G}^{(0)}$, with $s(D) \cup r(D) \subseteq C$, one has that $D C=D=C D$, so

$$
1_{D} 1_{C}=1_{D}=1_{C} 1_{D} .
$$

\footnotetext{
${ }^{3}$ Steinberg algebras may also be defined for ample groupoids that are not Hausdorff [22, Definition 4.1], but not as a straightforward generalization of the above.
} 
Thus, if we are given elements

$$
f_{i}=\sum_{D \in F_{i}} a_{D} 1_{D} \in A_{R}(\mathcal{G}), \quad 1 \leq i \leq n,
$$

where the $F_{i}$ are finite sets of compact open bissections, we may take

$$
C=\bigcup_{1 \leq i \leq n} \bigcup_{D \in F_{i}} s(D) \cup r(D),
$$

and it will follow that $1_{C}$ is an idempotent element of $A_{R}\left(\mathcal{G}^{(0)}\right)$, and

$$
f_{i} 1_{C}=f_{i}=1_{C} f_{i}, \quad \forall i=1, \ldots, n .
$$

An immediate consequence of the above result is that

$$
A_{R}(\mathcal{G})=A_{R}\left(\mathcal{G}^{(0)}\right) A_{R}(\mathcal{G})=A_{R}(\mathcal{G}) A_{R}\left(\mathcal{G}^{(0)}\right) .
$$

Recall that a subset $U \subseteq \mathcal{G}^{(0)}$ is said to be invariant if for every $\gamma$ in $\mathcal{G}$ one has that

$$
r(\gamma) \in U \Longleftrightarrow s(\gamma) \in U
$$

Given such an invariant subset $U$, let

$$
\begin{aligned}
\left.\mathcal{G}\right|_{U} & =\{\gamma \in \mathcal{G}: r(\gamma) \in U\} \\
& =\{\gamma \in \mathcal{G}: s(\gamma) \in U\}
\end{aligned}
$$

and observe that $\left.\mathcal{G}\right|_{U}$ is a subgroupoid of $\mathcal{G}$. If, in addition, $U$ is an open subset of $\mathcal{G}^{(0)}$, then $\left.\mathcal{G}\right|_{U}$ is open in $\mathcal{G}$. Therefore, as in $(2.5)$, we have that $A_{R}\left(\left.\mathcal{G}\right|_{U}\right)$ is a subalgebra of $A_{R}(\mathcal{G})$.

Regardless of whether or not $U$ is invariant, as long as it is an open subset of $\mathcal{G}^{(0)}$ we may always see $U$ itself as an open subgroupoid of $\mathcal{G}$. So, again by (2.5), we may identify $A_{R}(U)$ as a subalgebra of $A_{R}(\mathcal{G})$, observing that $A_{R}(U)$ is nothing but $C_{c}(R, U)$ with pointwise multiplication.

Given an algebra $A$, and given two subsets $X$ and $Y$ of $A$, we will denote by $X Y$ the $R$-submodule of $A$ generated by the set of products, namely

$$
X Y=\left\{\sum_{i=1}^{n} r_{i} x_{i} y_{i}: n \in \mathbb{N}, r_{1}, \ldots, r_{n} \in R, x_{1}, \ldots, x_{n} \in X, y_{1}, \ldots, y_{n} \in Y\right\} .
$$

This notation is used in the next:

Proposition 2.9. Let $\mathcal{G}$ be an ample, Hausdorff groupoid. Given an open subset $U \subseteq \mathcal{G}^{(0)}$, one has that $U$ is invariant in the sense of (2.8) if and only if

$$
A_{R}(\mathcal{G}) A_{R}(U)=A_{R}(U) A_{R}(\mathcal{G})
$$

In this case, one has that

$$
A_{R}\left(\left.\mathcal{G}\right|_{U}\right)=A_{R}(\mathcal{G}) A_{R}(U)=A_{R}(U) A_{R}(\mathcal{G}),
$$

and consequently $A_{R}\left(\left.\mathcal{G}\right|_{U}\right)$ is an ideal in $A_{R}(\mathcal{G})$.

Proof. Supposing that $U$ is invariant, let us first prove that $A_{R}(\mathcal{G}) A_{R}(U) \subseteq A_{R}\left(\left.\mathcal{G}\right|_{U}\right)$. For this it suffices to show that, given any compact open bissection $B \subseteq \mathcal{G}$, and any compact open subset $K \subseteq U$, one has that $1_{B} 1_{K} \in A_{R}\left(\left.\mathcal{G}\right|_{U}\right)$. To see this, notice that $1_{B} 1_{K}=1_{B K}$, as seen in (2.4), and that if $\gamma$ is any element of $\mathcal{G}$ lying in $B K$, then $s(\gamma) \in K \subseteq U$, whence $\left.\gamma \in \mathcal{G}\right|_{U}$. This shows that $\left.B K \subseteq \mathcal{G}\right|_{U}$, so $1_{B K}$ indeed belongs to $A_{R}\left(\left.\mathcal{G}\right|_{U}\right)$. 
Let us now prove that $A_{R}(\mathcal{G}) A_{R}(U) \supseteq A_{R}\left(\left.\mathcal{G}\right|_{U}\right)$. Again we may focus on characteristic functions, meaning that all we must do is show that $1_{B}$ lies in $A_{R}(\mathcal{G}) A_{R}(U)$, for all compact open bissections $\left.B \subseteq \mathcal{G}\right|_{U}$. Given such a $B$, observe that $s(B) \subseteq U$, so $1_{s(B)} \in A_{R}(U)$, and

$$
1_{B}=1_{B s(B)}=1_{B} 1_{s(B)} \in A_{R}(\mathcal{G}) A_{R}(U) .
$$

This proves that $A_{R}\left(\left.\mathcal{G}\right|_{U}\right)=A_{R}(\mathcal{G}) A_{R}(U)$, and one may similarly prove that $A_{R}\left(\left.\mathcal{G}\right|_{U}\right)=$ $A_{R}(U) A_{R}(\mathcal{G})$, which readily implies that $A_{R}\left(\left.\mathcal{G}\right|_{U}\right)$ is an ideal in $A_{R}(\mathcal{G})$.

In order to complete the proof we must show that the first identity displayed in the statement is a sufficient condition for the invariance of $U$. So, pick any $\gamma \in \mathcal{G}$, such that $s(\gamma) \in U$, and let us prove that $r(\gamma) \in U$, as well. For this, choose a compact open bissection $B$ containing $\gamma$. Replacing $B$ with $B K$, where $K$ is any compact open set such that

$$
s(\gamma) \in K \subseteq U
$$

we may suppose that $s(B) \subseteq U$. It follows that

$$
1_{B}=1_{B} 1_{s(B)} \in A_{R}(\mathcal{G}) A_{R}(U)=A_{R}(U) A_{R}(\mathcal{G}) .
$$

Since $A_{R}(U)$ has local units by (2.6), there exists an idempotent element in $A_{R}(U)$, necessarily of the form $1_{V}$, for some compact open set $V \subseteq U$, such that $1_{B}=1_{V} 1_{B}$. It follows that $B=V B$, and hence

$$
r(\gamma) \in r(B)=r(V B) \subseteq V \subseteq U .
$$

Similarly one proves that " $r(\gamma) \in U \Rightarrow s(\gamma) \in U$ ", so $U$ is invariant.

Given an ample, Hausdorff groupoid $\mathcal{G}$, for $u \in \mathcal{G}^{(0)}$, we let $\mathcal{G}_{u}^{u}$ denote the isotropy group at $u$. The isotropy group bundle is the set

$$
\operatorname{Iso}(\mathcal{G}):=\bigcup_{u \in \mathcal{G}^{(0)}} \mathcal{G}_{u}^{u} .
$$

We write Iso $(\mathcal{G})^{\circ}$ for the interior of the isotropy group bundle in the relative topology. Since $\mathcal{G}^{(0)}$ is open, one has that $\mathcal{G}^{(0)} \subseteq \operatorname{Iso}(\mathcal{G})^{\circ}$.

This gives us two canonical examples of open subgroupoids of $\mathcal{G}$, namely $\mathcal{G}^{(0)}$ and $\operatorname{Iso}(\mathcal{G})^{\circ}$. In the spirit of $(2.5)$ we then have

$$
A_{R}\left(\mathcal{G}^{(0)}\right) \subseteq A_{R}\left(\operatorname{Iso}(\mathcal{G})^{\circ}\right) \subseteq A_{R}(\mathcal{G})
$$

We say $\mathcal{G}$ is principal if $\operatorname{Iso}(\mathcal{G})=\mathcal{G}^{(0)}$. One can show this is equivalent to saying that

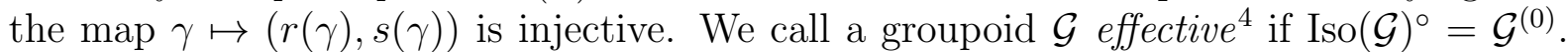
However, if $C$ is a closed invariant subset of $\mathcal{G}^{(0)}$, the restriction $\left.\mathcal{G}\right|_{C}$ might not be effective, even if $\mathcal{G}$ has this property. We therefore say that $\mathcal{G}$ is strongly effective if $\left.\mathcal{G}\right|_{C}$ is effective for every closed invariant subset $C \subseteq \mathcal{G}^{(0)}$.

\footnotetext{
${ }^{4}$ This has also been called essentially principal in a number of papers and that term is also somewhat standard. However, there are papers where essentially principal is used to mean something different so we have chosen to use 'effective' to avoid confusion.
} 


\section{A General uniqueness theorem}

In this section, we prove a generalised uniqueness theorem for the Steinberg algebra $A_{R}(\mathcal{G})$ associated to an ample, Hausdorff groupoid. Our theorem is the algebraic analogue of $\left[6\right.$, Theorem 3.1(c)] for groupoid $C^{*}$-algebras.

Theorem 3.1 (Generalized Uniqueness Theorem). Let $\mathcal{G}$ be a second-countable, ample, Hausdorff groupoid and let $R$ be a unital commutative ring. Suppose that $A$ is an $R$ algebra and that $\pi: A_{R}(\mathcal{G}) \rightarrow A$ is a ring homomorphism. Then $\pi$ is injective if and only if $\pi \circ \iota$ is injective.

We summarize the results we use to prove Theorem 3.1 in the following two lemmas.

Lemma 3.2 ([6, Lemma 3.3(a)]). Let $\mathcal{G}$ be a second-countable, ample, Hausdorff groupoid. Then

is dense in $\mathcal{G}^{(0)}$.

$$
X:=\left\{u \in \mathcal{G}^{(0)}: \mathcal{G}_{u}^{u} \subseteq \operatorname{Iso}(\mathcal{G})^{\circ}\right\}
$$

The next lemma is the algebraic analogue of [6, Lemma 3.3(b)]. We check that it holds in a purely algebraic setting.

Lemma 3.3. Let $\mathcal{G}$ be a second-countable, ample, Hausdorff groupoid and let $R$ be a unital commutative ring. Suppose $u \in \mathcal{G}^{(0)}$ is such that $\mathcal{G}_{u}^{u} \subseteq \operatorname{Iso}(\mathcal{G})^{\circ}$ and take $f \in A_{R}(\mathcal{G})$ such that there exists $\gamma_{u} \in \mathcal{G}_{u}^{u}$ with $f\left(\gamma_{u}\right) \neq 0$. Then there exists a compact open set $K \subseteq \mathcal{G}^{(0)}$ such that $u \in K$ and

$$
0 \neq 1_{K} f 1_{K} \in \iota\left(A_{R}\left(\operatorname{Iso}(\mathcal{G})^{\circ}\right)\right) .
$$

Proof. Write $f=\sum_{D \in F} a_{D} 1_{D}$ where $D$ is a disjoint collection of compact open bissections. For each $D \in F$, choose a compact open neighbourhood $V_{D} \subseteq \mathcal{G}^{(0)}$ as follows:

- If $u=r(\gamma)=s(\gamma)$ for some $\gamma \in D$, then $\gamma \in \operatorname{Iso}(\mathcal{G})^{\circ}$ by assumption. Let $V_{D}$ be a compact open subset in $\mathcal{G}^{(0)}$ containing $u$ such that $V_{D}$ is contained in the open set

$$
r\left(D \cap \operatorname{Iso}(\mathcal{G})^{\circ}\right)=s\left(D \cap \operatorname{Iso}(\mathcal{G})^{\circ}\right) .
$$

Then $V_{D} D V_{D} \subseteq D \cap \operatorname{Iso}(\mathcal{G})^{\circ}$.

- If there exists $\gamma \in D$ such that $r(\gamma)=u$ and $s(\gamma) \neq u$ or $s(\gamma)=u$ and $r(\gamma) \neq u$, we chose $V_{D}$ as follows. Because $\mathcal{G}$ is Hausdorff, we can find a compact open subset $D^{\prime} \subseteq D$ containing $\gamma$ such that $r\left(D^{\prime}\right) \cap s\left(D^{\prime}\right)=\emptyset$. Take $V_{D}=r\left(D^{\prime}\right)$ (or $\left.V_{D}=s\left(D^{\prime}\right)\right)$, so that $u \in V_{D}$ and $V_{D} D V_{D}=\emptyset$.

- If $u \notin r(D)$ and $u \notin s(D)$, use that $\mathcal{G}$ is Hausdorff to choose a neighbourhood $V_{D}$ of $u$ such that $V_{D} D V_{D}=\emptyset$.

Let

$$
K:=\bigcap_{D \in F} V_{D}
$$

Then $K$ is a compact open set that contains $u$ and by construction

$$
1_{K} f 1_{K} \in \iota\left(A_{R}\left(\operatorname{Iso}(\mathcal{G})^{\circ}\right)\right) .
$$

Furthermore

$$
1_{K} f 1_{K}\left(\gamma_{u}\right)=1_{K}\left(r\left(\gamma_{u}\right)\right) f\left(\gamma_{u}\right) 1_{K}\left(s\left(\gamma_{u}\right)\right)=f\left(\gamma_{u}\right) \neq 0,
$$

so $1_{K} f 1_{K} \neq 0$. 
Proof of Theorem 3.1. We show that $\pi$ not injective implies $\pi \circ \iota$ not injective. Suppose ker $\pi \neq\{0\}$. By [8, Lemma 3.1] there exists a function $f \in \operatorname{ker} \pi$ such that $\operatorname{supp} f \cap \mathcal{G}^{(0)} \neq$ $\emptyset$. Because $\mathcal{G}$ is Hausdorff, supp $f \cap \mathcal{G}^{(0)}$ is an open subset of $\mathcal{G}^{(0)}$. Thus Lemma 3.2 implies that there exists $u \in \operatorname{supp} f \cap \mathcal{G}^{(0)}$ such that $\mathcal{G}_{u}^{u} \subseteq \operatorname{Iso}(\mathcal{G})^{\circ}$. Now apply Lemma 3.3 (with $\gamma_{u}=u$ ) to get a nonempty compact open set $K \subseteq \mathcal{G}^{(0)}$ such that

$$
\operatorname{supp}\left(1_{K} f 1_{K}\right) \subseteq \iota\left(A_{R}\left(\operatorname{Iso}(\mathcal{G})^{\circ}\right)\right) .
$$

Since ker $\pi$ is an ideal, we also have $1_{K} f 1_{K} \in \operatorname{ker} \pi$. Thus $0 \neq 1_{K} f 1_{K} \in \operatorname{ker}(\pi \circ \iota)$.

In Corollary 3.4 below, we see that the usual Cuntz-Krieger uniqueness theorem (see [8, Theorem 3.2] or [23, Proposition 3.3]) for effective ample groupoids is a consequence of Theorem 3.1. In particular, if $\mathcal{G}$ is effective, then $\operatorname{Iso}(\mathcal{G})^{\circ}=\mathcal{G}^{(0)}$. Thus $\pi \circ \iota$ is injective if and only if $\pi\left(r 1_{K}\right) \neq 0$ for all compact $K \subseteq \mathcal{G}^{(0)}$ and $r \in R \backslash\{0\}$.

Corollary 3.4 (The Cuntz-Krieger Uniqueness Theorem). Let $\mathcal{G}$ be an effective, secondcountable, ample, Hausdorff groupoid and let $R$ be a unital commutative ring. Suppose $A$ is an $R$-algebra and $\pi: A_{R}(\mathcal{G}) \rightarrow A$ is a ring homomorphism. Then $\pi$ is injective if and only if $\pi\left(r 1_{K}\right) \neq 0$ for all compact open subset $K \subseteq \mathcal{G}^{(0)}$ and $r \in R \backslash\{0\}$.

In [13, Proposition 2.3] it is shown that the Cuntz-Krieger uniqueness theorem is false for $C^{*}$-algebras associated to non-Hausdorff groupoids. Similarly, it is false in for the Steinberg algebras associated to ample, non-Hausdorff groupoids. Although both Lemma 3.2 and Lemma 3.3 hold in the non-Hausdorff setting, the following slight modification of [13, Proposition 2.3] illustrates that the uniqueness theorem itself fails.

Example 3.5. Let $\mathcal{G}$ be the groupoid described in [13, Section 2], and recall that the unit space of $\mathcal{G}$ is the subset $Z \subseteq \mathbb{R}^{2}$ obtained as the union of the sets

$$
X=[-1,1] \times\{0\}, \quad \text { and } Y=\{0\} \times[-1,1] .
$$

Let $L$ be the subset of $[-1,1]$ given by

$$
L=\{ \pm 1 / n: n \in \mathbb{N}, n \geq 1\} \cup\{0\},
$$

and put

$$
X^{\prime}=L \times\{0\}, \quad \text { and } \quad Y^{\prime}=\{0\} \times L .
$$

Then $Z^{\prime}:=X^{\prime} \cup Y^{\prime}$ is easily seen to be a closed invariant subset of $Z$, so

$$
\mathcal{G}^{\prime}:=\left.\mathcal{G}\right|_{Z^{\prime}}
$$

is an étale subgroupoid of $\mathcal{G}$. Observing that $Z^{\prime}$ is zero-dimensional, we have that $\mathcal{G}^{\prime}$ is an ample groupoid and it is not hard to see that it is also effective.

If we replace $\mathcal{G}$ by $\mathcal{G}^{\prime}$, and $f$ by its restriction to $\mathcal{G}^{\prime}$ in [13, Proposition 2.2], then it is clear that the conclusion of this result still holds, and hence, as in [13, Proposition 2.3], one has that $\mathbb{C} f$ is a nontrivial ideal in $A_{\mathbb{C}}\left(\mathcal{G}^{\prime}\right)$ trivially intersecting $A_{\mathbb{C}}\left(\mathcal{G}^{\prime(0)}\right)$.

\section{IDEALS IN GRADED ALGEBRAS}

Our next major goal will be to study graded ideals in Steinberg algebras. In preparation for this we will now discuss certain aspects of ideals in abstract graded algebras. Throughout this section we will fix a unital commutative ring $R$. All algebras in this section will be understood to be $R$-algebras. 
Definition 4.1. We will say that an algebra $A$ is graded over a given group $\Gamma$, or that $A$ is $\Gamma$-graded, if $A$ is equipped with a direct sum decomposition

$$
A=\bigoplus_{g \in \Gamma} A_{g}
$$

where each $A_{g}$ is an $R$-submodule, satisfying

$$
A_{g} A_{h} \subseteq A_{g h}, \quad \forall g, h \in \Gamma .
$$

In this case we will say each $A_{g}$ is the homogeneous submodule associated to the group element $g$.

The central concept to be studied in this section is defined next.

Definition 4.2. Let $A$ be a $\Gamma$-graded algebra. An ideal $J \unlhd A$ is said to be a graded ideal if $J=\bigoplus_{g \in \Gamma} J_{g}$, where each $J_{g}=J \cap A_{g}$.

It is an elementary exercise to show that $J$ is graded if and only if $\pi_{g}(J) \subseteq J$, for every $g$ in $G$, where $\pi_{g}$ is the standard projection of $A$ onto $A_{g}$.

Definition 4.3. Given a $\Gamma$-graded algebra, we will say that an ideal $I \unlhd A_{e}$ is invariant provided

$$
A_{g} I=I A_{g}, \quad \forall g \in \Gamma .
$$

Invariant ideals give rise to graded ideals as follows:

Proposition 4.4. Given a $\Gamma$-graded algebra, and an invariant ideal $I \unlhd A_{e}$, one has that

$$
\operatorname{Ind}(I)=\bigoplus_{g \in \Gamma} I A_{g}
$$

is a graded ideal in $A$, henceforth called the ideal induced by $I$.

Proof. Left to the reader.

It would be nice if invariant ideals in $A_{e}$ were in a bijective correspondence with graded ideals in $A$, according to the above procedure, but this is not always true, unless we restrict ourselves to special situations which we now describe.

In order to introduce our first major condition, observe that the polynomial algebra $R[X]$ is a $\mathbb{Z}$-graded algebra; the homogeneous submodule associated to a nonnegative integer $n$ is the set of all monomials $a X^{n}$, for $a$ in $R$, while all other homogeneous submodules are zero. This disparity between homogeneous submodules for positive and negative integers is an undesirable feature of this graded algebra which we will rule out via the following:

Definition 4.5. We will say that the graded algebra $A=\bigoplus_{g \in \Gamma} A_{g}$, is symmetrically graded if

for each $g$ in $\Gamma$.

$$
A_{g} A_{g^{-1}} A_{g}=A_{g}
$$

Observe that a necessary condition for $A=\bigoplus_{g \in \Gamma} A_{g}$ to be a symmetrically graded algebra is that $A_{e}=A_{e}^{3}$, which implies that

$$
A_{e}=A_{e}^{3} \subseteq A_{e}^{2} \subseteq A_{e},
$$

so that $A_{e}=A_{e}^{2}$, which is to say that $A_{e}$ is an idempotent algebra. 
Speaking of graded ideals, observe that these may also be considered as graded algebras which may or may not be symmetrically graded. For the case of induced ideals we have:

Proposition 4.6. Given a symmetrically $\Gamma$-graded algebra $A$, and an invariant ideal $I \unlhd A_{e}$, consider the following statements:

(a) I is idempotent,

(b) $\operatorname{Ind}(I)$ is symmetrically graded.

Then (a) implies (b) and the converse holds provided $A_{e}$ is s-unital.

Proof. Set $J=\operatorname{Ind}(I)$, so $J_{g}=J \cap A_{g}=I A_{g}$. Assuming $(a)$, we then have

$$
J_{g} J_{g^{-1}} J_{g}=I A_{g} I A_{g^{-1}} I A_{g}=I^{3} A_{g} A_{g^{-1}} A_{g}=I A_{g}=J_{g}
$$

so $J$ is symmetrically graded. Conversely, assuming $(b)$, we have already seen that $J_{e}$ is idempotent. Moreover, since $A_{e}$ is $s$-unital we have

$$
I=A_{e} I=J_{e}
$$

from where $(a)$ follows.

The above result may be seen as a process to produce symmetrically graded ideals in $A$ out of invariant idempotent ideals of $A_{e}$, and we will now prove that all such ideals arise from this construction.

Proposition 4.7. Given a $\Gamma$-graded algebra, and a graded ideal $J \unlhd A$, suppose that $J$ is symmetrically graded. Then $J_{e}=J \cap A_{e}$ is an invariant idempotent ideal of $A_{e}$, and $J=\operatorname{Ind}\left(J_{e}\right)$.

Proof. Setting $J_{g}=J \cap A_{g}$, we have

$$
J_{e} A_{g}=\left(J \cap A_{e}\right) A_{g} \subseteq J \cap A_{g}=J_{g}=J_{g} J_{g^{-1}} J_{g} \subseteq J_{e} J_{g} \subseteq J_{e} A_{g},
$$

from where we see that $J_{e} A_{g}=J_{g}$ and one may similarly prove that $A_{g} J_{e}=J_{g}$. In particular $J_{e} A_{g}=A_{g} J_{e}$, so $J_{e}$ is invariant and clearly idempotent. Moreover, since $J$ is graded, we have

$$
J=\bigoplus_{g \in \Gamma} J_{g}=\bigoplus_{g \in \Gamma} J_{e} A_{g}=\operatorname{Ind}\left(J_{e}\right) .
$$

Aided by our last results we may now prove:

Proposition 4.8. Let $A=\bigoplus_{g \in \Gamma} A_{g}$ be a symmetrically graded algebra such that $A_{e}$ is s-unital. Then the correspondence

$$
I \mapsto \operatorname{Ind}(I)
$$

is a bijection from the set of all invariant idempotent ideals $I \unlhd A_{e}$ onto the set of all symmetrically graded ideals $J \unlhd A$.

Proof. Given an invariant idempotent ideal $I \unlhd A_{e}$, we have by (4.6) that $\operatorname{Ind}(I)$ is indeed a symmetrically graded ideal, hence our correspondence is well defined. It is moreover surjective by (4.7).

Given any invariant ideal $I \unlhd A_{e}$, observe that

$$
\operatorname{Ind}(I) \cap A_{e}=I A_{e}=I,
$$

where the last equality is a consequence of the fact that $A_{e}$ is $s$-unital. If $I^{\prime}$ is another invariant ideal with $\operatorname{Ind}(I)=\operatorname{Ind}\left(I^{\prime}\right)$, then

$$
I=\operatorname{Ind}(I) \cap A_{e}=\operatorname{Ind}\left(I^{\prime}\right) \cap A_{e}=I^{\prime} .
$$


This shows that Ind is one-to-one, so our proof is concluded.

\section{Graded ideals in Steinberg ALGeBras}

Throughout this section $\mathcal{G}$ will always refer to an ample, Hausdorff groupoid. Given a group $\Gamma$, recall that a map

$$
c: \mathcal{G} \rightarrow \Gamma,
$$

is said to be a cocycle if $c\left(\gamma_{1} \gamma_{2}\right)=c\left(\gamma_{1}\right) c\left(\gamma_{2}\right)$, for every $\left(\gamma_{1}, \gamma_{2}\right) \in \mathcal{G}^{(2)}$. Cocycles are sometimes also called homomorphisms. Given such a cocycle, for each $g \in \Gamma$, we shall denote by

$$
\mathcal{G}_{g}=\{\gamma \in \mathcal{G}: c(\gamma)=g\} .
$$

Assuming that $c$ is locally constant (which is to say that $c$ is continuous when $\Gamma$ is given the discrete topology), observe that each $\mathcal{G}_{g}$ is an open set. We may then view $C_{c}\left(\mathcal{G}_{g}, R\right)$ as a submodule of $A_{R}(\mathcal{G})$, as in (2.1).

Proposition 5.1. Given an ample, Hausdorff groupoid $\mathcal{G}$, and a locally constant $\Gamma$-valued cocycle c on $\mathcal{G}$, let

$$
A_{g}=C_{c}\left(\mathcal{G}_{g}, R\right), \quad \forall g \in \Gamma .
$$

Then the $A_{g}$ are homogeneous submodules for a grading of $A_{R}(\mathcal{G})$, which is moreover a symmetrical grading.

Proof. We leave it for the reader to verify that we indeed have a grading. In order to prove symmetry, let $B$ be a compact open bissection in $\mathcal{G}_{g}$. Then $B^{-1} \subseteq \mathcal{G}_{g^{-1}}$, so $1_{B^{-1}} \subseteq A_{g^{-1}}$. Moreover, one has

$$
1_{B}=1_{B} 1_{B^{-1}} 1_{B} \in A_{g} A_{g^{-1}} A_{g} .
$$

Since $A_{g}$ is spanned by the elements of the form $1_{B}$, with $B$ as above, we conclude that

$$
A_{g} \subseteq A_{g} A_{g^{-1}} A_{g}
$$

and, since the reverse inclusion is clearly true, the proof is complete.

The fact that $A_{R}(\mathcal{G})$ is symmetrically graded does not necessarily imply that all of its graded ideals share that property. However in the following special situation they do:

Lemma 5.2. Let $K$ be a field ${ }^{5}, \mathcal{G}$ be an ample, Hausdorff groupoid, and let $c$ be a locally constant $\Gamma$-valued cocycle on $\mathcal{G}$. If $c^{-1}(e)$ is a strongly effective groupoid, then every graded ideal of $A_{K}(\mathcal{G})$ is symmetrically graded.

Proof. Let $J$ be a graded ideal in $A_{K}(\mathcal{G})$. Setting $J_{g}=J \cap A_{g}$, our task is to prove that

$$
J_{g}=J_{g} J_{g^{-1}} J_{g}, \quad \forall g \in \Gamma \text {. }
$$

We leave the trivial inclusion " $J_{g} \supseteq J_{g} J_{g^{-1}} J_{g}$ " for the reader to verify, and concentrate on the opposite one. Given $g$ in $\Gamma$, pick any $f \in J_{g}$, and write

$$
f=\sum_{D \in F} a_{D} 1_{D}
$$

where $F$ is a finite collection of pairwise disjoint compact open bissections and the $a_{D}$ are nonzero elements of $K$. By [8, Lemma 2.2], we may suppose, without loss of generally, that each $D$ in $F$ is contained in a single $\mathcal{G}_{h}$.

\footnotetext{
${ }^{5}$ From now on we will need our $\operatorname{ring} R$ to be a field. In this setting we write $K$ instead of $R$.
} 
Denoting by $\pi_{g}$ the canonical projection from $A_{K}(\mathcal{G})$ to $A_{g}$, we then have

$$
f=\pi_{g}(f)=\sum_{D \in F} a_{D} \pi_{g}\left(1_{D}\right)
$$

Notice that if $D$ is contained in $\mathcal{G}_{h}$, then $1_{D}$ lies in $A_{h}$. So $\pi_{g}\left(1_{D}\right)=1_{D}$, when $g=h$, while $\pi_{g}\left(1_{D}\right)=0$, otherwise. Consequently we have

$$
f=\sum_{\substack{D \in F \\ D \subseteq \mathcal{G}_{g}}} a_{D} 1_{D}
$$

which amounts to saying that, in the original description $(\dagger)$ of $f$, we may assume that $D \subseteq \mathcal{G}_{g}$, for every $D$ in $F$.

Our next goal will be to prove that $1_{D}$ lies in $J$, for each $D$ in $F$. Given $D_{0}$ in $F$, set $f^{\prime}=1_{D_{0}^{-1}} f$. Observing that $1_{D_{0}^{-1}}$ lies in $A_{g^{-1}}$, we have

$$
f^{\prime}=1_{D_{0}^{-1}} f \in A_{g^{-1}} A_{g} \subseteq A_{e}
$$

and it is clear that $f^{\prime}$ also lies in $J$, so $f^{\prime} \in J_{e}$.

Recalling that $\mathcal{G}_{e}$ is a strongly effective groupoid, we may employ [9, Lemma 4.3] for the ideal

$$
J_{e} \unlhd A_{e}=A_{K}\left(\mathcal{G}_{e}\right),
$$

leading up to the conclusion that $\left.f^{\prime}\right|_{\mathcal{G}^{(0)}} \in J_{e}$. If $D$ is any member of $F$ other than $D_{0}$, hence disjoint from $D_{0}$, it is easy to see that $D_{0}^{-1} D$ does not intercept $\mathcal{G}^{(0)}$, so

$$
\left.f^{\prime}\right|_{\mathcal{G}^{(0)}}=\left.\left(\sum_{D \in F} a_{D} 1_{D_{0}^{-1}} 1_{D}\right)\right|_{\mathcal{G}^{(0)}}=\left.\left(\sum_{D \in F} a_{D} 1_{D_{0}^{-1} D}\right)\right|_{\mathcal{G}^{(0)}}=a_{D_{0}} 1_{D_{0}^{-1} D_{0}} .
$$

From this we deduce that

$$
1_{D_{0}}=\left(a_{D_{0}}^{-1} 1_{D_{0}}\right)\left(a_{D_{0}} 1_{D_{0}^{-1} D_{0}}\right)=\left(a_{D_{0}}^{-1} 1_{D_{0}}\right)\left(\left.f^{\prime}\right|_{\mathcal{G}^{(0)}}\right) \in J
$$

This proves our claim that $1_{D}$ lies in $J$, for every $D$ in $F$, and observe that $1_{D^{-1}}$ also lies in $J$ because

$$
1_{D^{-1}}=1_{D^{-1}} 1_{D} 1_{D^{-1}} \in A_{K}(\mathcal{G}) J A_{K}(\mathcal{G}) \subseteq J
$$

Since $D \subseteq \mathcal{G}_{g}$, and hence $D^{-1} \subseteq \mathcal{G}_{g^{-1}}$, we have that $1_{D} \in J_{g}$, and $1_{D^{-1}} \in J_{g^{-1}}$, so

$$
1_{D}=1_{D} 1_{D^{-1}} 1_{D} \in J_{g} J_{g^{-1}} J_{g}
$$

from where it follows that $f$ also lies in $J_{g} J_{g^{-1}} J_{g}$, thus proving that

$$
J_{g} \subseteq J_{g} J_{g^{-1}} J_{g}
$$

With this we may prove the following main result:

Theorem 5.3. Let $K$ be a field, $\mathcal{G}$ be an ample, Hausdorff groupoid, $\Gamma$ be a discrete group, and $c: \mathcal{G} \rightarrow \Gamma$ be a locally constant cocycle such that $c^{-1}(e)$ is strongly effective. Then the correspondence

$$
U \mapsto A_{K}\left(\left.\mathcal{G}\right|_{U}\right)
$$

is an isomophism from the lattice of open invariant subsets of $\mathcal{G}^{(0)}$ onto to the lattice of graded ideals in $A_{K}(\mathcal{G})$. 
Proof. Given any $f$ in $A_{K}(\mathcal{G})$, and any $g$ in $\Gamma$, observe that the homogeneous component $f_{g}$ of $f$ is obtained by taking the restriction of $f$ to the clopen set $\mathcal{G}_{g}$. If $f$ is in $A_{K}\left(\left.\mathcal{G}\right|_{U}\right)$, where $U$ is a given open invariant subset of $\mathcal{G}^{(0)}$, it is then clear that $f_{g}$ is also in $A_{K}\left(\left.\mathcal{G}\right|_{U}\right)$, so $A_{K}\left(\left.\mathcal{G}\right|_{U}\right)$ is indeed a graded ideal. Therefore the correspondence mentioned in the statement is well defined.

Let $J$ be a graded ideal of $A_{K}(\mathcal{G})$. Then by (4.8), (5.1), (2.6) and (5.2), there is an invariant ideal

$$
I \unlhd A_{e}=A_{K}\left(\mathcal{G}_{e}\right),
$$

such that $J=\operatorname{Ind}(I)$. Since $\mathcal{G}_{e}$ is assumed to be strongly effective, we have by [9, Theorem 3.1] that there is an open subset $U \subseteq \mathcal{G}^{(0)}$, which is invariant relative to $\mathcal{G}_{e}$, and such that $I$ consists of all $f$ in $A_{K}\left(\mathcal{G}_{e}\right)$ whose support lies in $\left.\mathcal{G}_{e}\right|_{U}$. In other words $I=A_{K}\left(\left.\mathcal{G}_{e}\right|_{U}\right)$.

We next claim that the invariance of $I$ (in the sense of 4.3) implies that $U$ is in fact invariant relative to $\mathcal{G}$ (in the sense of 2.8). In order to check this we will resort to (2.9), so it suffices to prove that $A_{K}(\mathcal{G}) A_{K}(U)=A_{K}(U) A_{K}(\mathcal{G})$. Observing that $\mathcal{G}^{(0)} \subseteq \mathcal{G}_{e}$, we have by (2.7) that

$$
A_{K}(\mathcal{G})=A_{K}(\mathcal{G}) A_{K}\left(\mathcal{G}_{e}\right)=A_{K}\left(\mathcal{G}_{e}\right) A_{K}(\mathcal{G})
$$

so

$$
\begin{aligned}
& A_{K}(\mathcal{G}) A_{K}(U)=A_{K}(\mathcal{G}) A_{K}\left(\mathcal{G}_{e}\right) A_{K}(U) \stackrel{(2.9)}{=} A_{K}(\mathcal{G}) A_{K}\left(\left.\mathcal{G}_{e}\right|_{U}\right)=A_{K}(\mathcal{G}) I= \\
& =I A_{K}(\mathcal{G})=A_{K}\left(\left.\mathcal{G}_{e}\right|_{U}\right) A_{K}(\mathcal{G})=A_{K}(U) A_{K}\left(\mathcal{G}_{e}\right) A_{K}(\mathcal{G})=A_{K}(U) A_{K}(\mathcal{G})
\end{aligned}
$$

verifying the appropriate hypothesis in (2.9), and hence proving our claim that $U$ is invariant relative to $\mathcal{G}$.

We next observe that

$$
\begin{gathered}
J=\operatorname{Ind}(I)=\bigoplus_{g \in \Gamma} I A_{g}=I\left(\bigoplus_{g \in \Gamma} A_{g}\right)=I A_{K}(\mathcal{G})=A_{K}\left(\left.\mathcal{G}_{e}\right|_{U}\right) A_{K}(\mathcal{G})= \\
=A_{K}(U) A_{K}\left(\mathcal{G}_{e}\right) A_{K}(\mathcal{G})=A_{K}(U) A_{K}(\mathcal{G})=A_{K}\left(\left.\mathcal{G}\right|_{U}\right) .
\end{gathered}
$$

This shows that our correspondence is surjective. To see that it is also injective, let $U$ be an open invariant subset of $\mathcal{G}^{(0)}$ and observe that $\left.\mathcal{G}\right|_{U} \cap \mathcal{G}^{(0)}=U$, so

$$
A_{K}\left(\left.\mathcal{G}\right|_{U}\right) \cap A_{K}\left(\mathcal{G}^{(0)}\right)=A_{K}(U)
$$

This says that $A_{K}(U)$, and hence also $U$, may be recovered from $A_{K}\left(\left.\mathcal{G}\right|_{U}\right)$, from where injectiveness follows.

We leave it for the reader to prove that the corresponding lattice structures are preserved.

\section{Example: Groups acting on graphs}

In this section, we consider the algebras $\mathcal{O}_{G, E}$ associated to triples $(G, E, \varphi)$, introduced in [17]. 
6.1. The algebra $\mathcal{O}_{G, E}$. Let us recall the construction.

6.1. The basic data for our construction is a triple $(G, E, \varphi)$ composed of:

(1) A finite directed graph $E=\left(E^{0}, E^{1}, r, s\right)$ without sources.

(2) A discrete group $G$ acting on $E$ by graph automorphisms.

(3) A 1-cocycle $\varphi: G \times E^{1} \rightarrow G$ satisfying the property

$$
\varphi(g, a) \cdot v=g \cdot v \text { for every } g \in G, a \in E^{1}, v \in E^{0} .
$$

The property (3) required on $\varphi$ is tagged (2.3) in [17].

Definition 6.2. Given a triple $(G, E, \varphi)$ as in (6.1), we define $\mathcal{O}_{G, E}$ to be the universal $C^{*}$-algebra as follows:

(1) Generators:

$$
\left\{p_{x}: x \in E^{0}\right\} \cup\left\{s_{a}: a \in E^{1}\right\} \cup\left\{u_{g}: g \in G\right\} .
$$

(2) Relations:

(a) $\left\{p_{x}: x \in E^{0}\right\} \cup\left\{s_{a}: a \in E^{1}\right\}$ is a Cuntz-Krieger $E$-family in the sense of [20].

(b) The map $u: G \rightarrow \mathcal{O}_{G, E}$ defined by the rule $g \mapsto u_{g}$ is a unitary *-representation of $G$.

(c) $u_{g} s_{a}=s_{g \cdot a} u_{\varphi(g, a)}$ for every $g \in G, a \in E^{1}$.

(d) $u_{g} p_{x}=p_{g \cdot x} u_{g}$ for every $g \in G, x \in E^{0}$.

Notice that the relation (2a) in Definition 6.2 implies that there is a natural representation map

$$
\begin{aligned}
\phi: C^{*}(E) & \rightarrow \mathcal{O}_{G, E} \\
p_{x} & \mapsto p_{x} \\
s_{a} & \mapsto s_{a}
\end{aligned}
$$

which is injective [17, Proposition 11.1].

6.2. The groupoid $\mathcal{G}_{(G, E)}$. Recall from [17, Definition 4.1] that given a triple $(G, E, \varphi)$ as in (6.1), we define a $*$-inverse semigroup $\mathcal{S}_{G, E}$ as follows:

(1) The set is

$$
\mathcal{S}_{G, E}=\left\{(\alpha, g, \beta): \alpha, \beta \in E^{*}, g \in G, d(\alpha)=g d(\beta)\right\} \cup\{0\},
$$

where $E^{*}$ denotes the set of finite paths in $E$.

(2) The operation is defined by:

$$
(\alpha, g, \beta) \cdot(\gamma, h, \delta):=\left\{\begin{array}{cl}
(\alpha, g \varphi(h, \varepsilon), \delta h \varepsilon), & \text { if } \beta=\gamma \varepsilon \\
(\alpha g \varepsilon, \varphi(g, \varepsilon) h, \delta), & \text { if } \gamma=\beta \varepsilon \\
0, & \text { otherwise }
\end{array}\right.
$$

and $(\alpha, g, \beta)^{*}:=\left(\beta, g^{-1}, \alpha\right)$.

Then, we can construct the groupoid of germs of the action of $\mathcal{S}_{G, E}$ on the compact space $\widehat{\mathcal{E}}$ of characters of the semilattice $\mathcal{E}$ of idempotents of $\mathcal{S}_{G, E}$. In our concrete case, $\widehat{\mathcal{E}}$ turns out to be homeomorphic to the compact space $E^{\infty}$ of one-sided infinite paths on $E$; the action of $(\alpha, g, \beta) \in \mathcal{S}_{G, E}$ on $\eta=\beta \widehat{\eta}$ is given by the rule $(\alpha, g, \beta) \cdot \eta=\alpha(g \widehat{\eta})$. Thus, the groupoid of germs is

$$
\mathcal{G}_{(G, E)}=\{[\alpha, g, \beta ; \eta]: \eta=\beta \widehat{\eta}\}
$$


where $[s ; \eta]=[t ; \mu]$ if and only if $\eta=\mu$ and there exists $0 \neq e^{2}=e \in \mathcal{S}_{G, E}$ such that $e \cdot \eta=\eta$ and $s e=t e$. The unit space

$$
\mathcal{G}_{(G, E)}{ }^{(0)}=\{[\alpha, 1, \alpha ; \eta]: \eta=\alpha \widehat{\eta}\}
$$

is identified with the one-sided infinite path space $E^{\infty}$, via the homeomorphism $[\alpha, 1, \alpha ; \eta] \mapsto \eta$. Under this identification, the range and source maps on $\mathcal{G}_{(G, E)}$ are:

$$
s([\alpha, g, \beta ; \beta \widehat{\eta}])=\beta \widehat{\eta} \quad \text { and } \quad r([\alpha, g, \beta ; \beta \widehat{\eta}])=\alpha(g \widehat{\eta}) .
$$

A basis for the topology on $\mathcal{G}_{(G, E)}$ is given by compact open bissections of the form

$$
\Theta(\alpha, g, \beta ; Z(\gamma)):=\left\{[\alpha, g, \beta ; \xi] \in \mathcal{G}_{(G, E)}: \xi \in Z(\gamma)\right\}
$$

where $\gamma \in E^{*}$ and $Z(\gamma):=\left\{\gamma \widehat{\eta}: \widehat{\eta} \in E^{\infty}\right\}$. Thus $\mathcal{G}_{(G, E)}$ is locally compact and ample. In [17] characterizations are given for when $\mathcal{G}_{(G, E)}$ is Hausdorff [17, Theorem 12.2], amenable [17, Corollary 10.18] or effective [17, Theorem 14.10] in terms of the properties of the triple $(G, E, \varphi)$ and the action of $\mathcal{S}_{G, E}$ on $E^{\infty}$ (see [16] for a version of these results in the case of tight groupoids of arbitrary inverse semigroups).

By [17, Theorem 6.3 \& Corollary 6.4], we have a *-isomorphism $\mathcal{O}_{G, E} \cong C^{*}\left(\mathcal{G}_{(G, E)}\right)$, so that $\mathcal{O}_{G, E}$ can be seen as a full groupoid $C^{*}$-algebra. The complex Steinberg algebra $A_{\mathbb{C}}\left(\mathcal{G}_{(G, E)}\right)$ is a dense subalgebra of $\mathcal{O}_{G, E} \cong C^{*}\left(\mathcal{G}_{(G, E)}\right)$ by [22, Proposition 6.7].

6.3. The Steinberg algebra $A_{R}\left(\mathcal{G}_{(G, E)}\right)$. Now, we will prove that for any unital commutative ring $R$, the Steinberg algebra $A_{R}\left(\mathcal{G}_{(G, E)}\right)$ is isomorphic to the $R$-algebra $\mathcal{O}_{(G, E)}^{\text {alg }}(R)$ with presentation given by Definition 6.2 (i.e. the algebraic version of $\mathcal{O}_{(G, E)}$ ).

Proposition 6.3. The map

$$
\begin{aligned}
& \pi: \quad S_{G, E} \rightarrow \mathcal{O}_{(G, E)}^{a l g}(R) \\
& (\alpha, g, \beta) \mapsto s_{\alpha} u_{g} s_{\beta}^{*}
\end{aligned}
$$

is the universal tight representation of $S_{G, E}$ in the category of $R$-algebras.

Proof. This is a direct consequence of the arguments used to prove [17, Proposition 6.2] and [17, Theorem 6.3].

Since $\mathcal{G}_{(G, E)}$ is the groupoid of germs for the natural action of $S_{G, E}$ on the space of tight filters over its idempotent semi-lattice, we can argue as in the proof of [15, Theorem 2.4] (see $[15,(2.4 .2)])$ to show that the map

$$
\begin{array}{cccc}
\phi: \quad A_{R}\left(\mathcal{G}_{(G, E)}\right) & \rightarrow & \mathcal{O}_{(G, E)}^{\mathrm{alg}}(R) \\
1_{\Theta((\alpha, g, \beta), Z(\beta))} & \mapsto & s_{\alpha} u_{g} s_{\beta}^{*}
\end{array}
$$

is a well-defined $R$-algebra homomorphism that is onto.

On the other side, the representation map

$$
\begin{array}{ccc}
\rho: \quad S_{G, E} & \rightarrow & A_{R}\left(\mathcal{G}_{(G, E)}\right) \\
(\alpha, g, \beta) & \mapsto 1_{\Theta((\alpha, g, \beta), Z(\beta))}
\end{array}
$$

is tight. Hence, the universal property stated in Proposition 6.3 says

$$
\begin{aligned}
\psi: \mathcal{O}_{(G, E)}^{\text {alg }}(R) & \rightarrow A_{R}\left(\mathcal{G}_{(G, E)}\right) \\
s_{\alpha} u_{g} s_{\beta}^{*} & \mapsto 1_{\Theta((\alpha, g, \beta), Z(\beta))}
\end{aligned}
$$

is an $R$-algebra homomorphism. Moreover, since $\widehat{\mathcal{E}\left(\widehat{S_{G, E}}\right)_{\text {tight }}}$ is Boolean [22], $\psi$ is onto $[22$, Proposition 5.13(7)]. Clearly, $\phi$ and $\psi$ are mutually inverses. So, we conclude 
Theorem 6.4. The map $\phi: A_{R}\left(\mathcal{G}_{(G, E)}\right) \rightarrow \mathcal{O}_{(G, E)}^{\text {alg }}(R)$ is an $R$-algebra isomorphism.

Notice that, through the isomorphism $\psi$, we can identify the Leavitt path algebra $L_{R}(E)$ of the graph $E$ (which is a subalgebra of $\mathcal{O}_{(G, E)}^{\text {alg }}(R)$ ) with a subalgebra of $A_{R}\left(\mathcal{G}_{(G, E)}\right)$ isomorphic to the Steinberg algebra $A_{R}\left(\mathcal{G}_{E}\right)$ of the path groupoid $\mathcal{G}_{E}$.

An interesting application of Theorem 6.4 is the following characterization of simplicity. First we give the relavent definitions from [17].

- We say $E$ is weakly $G$-transitive if, given any infinite path $\zeta$, and any vertex $x \in E^{0}$, there is some vertex $v$ along $\zeta$ such that there exists a vertex $u$ with $u=g x$ for some $g \in G$ and there is a path from $v$ to $u$.

- A $G$-circuit is a pair $(g, \gamma)$, where $g \in G$, and $\gamma \in E^{*}$ is a finite path of nonzero length such that $s(\gamma)=\operatorname{gr}(\gamma)$.

- Given a $G$-circuit $(g, \gamma)$ such that $\gamma=\gamma_{1} \gamma_{2} \ldots \gamma_{n}$ in $E^{*}$ and each $\gamma_{i}$ is in $E^{1}$, we say that $\gamma$ has no entry if $r^{-1}\left(s\left(\gamma_{i}\right)\right)$ is a singleton for every $i=1, \ldots, n$, i.e. $r^{-1}\left(s\left(\gamma_{i}\right)\right)=\left\{\gamma_{i+1}\right\}$ for every $i=1, \ldots, n-1$, and $r^{-1}\left(s\left(\gamma_{n}\right)\right)=\left\{g \gamma_{1}\right\}$.

- Given $g \in G$, and $x \in E^{0}$, we shall say that $g$ is slack at $x$, if there is a nonnegative integer $n$ such that all finite paths $\gamma$ with $r(\gamma)=x$, and $|\gamma| \geq n$, are strongly fixed by $g$, as defined in the sense that $g \gamma=\gamma$, and $\phi(g, \gamma)=1$.

Corollary 6.5. Suppose that $\mathcal{G}_{(G, E)}$ is Hausdorff. Then, for any field $K$ the following are equivalent:

(1) The algebra $\mathcal{O}_{G, E}^{\text {alg }}(K)$ is simple.

(2) The following properties hold:

(a) $E$ is weakly G-transitive.

(b) Every $G$-circuit has an entry.

(c) Given $x \in E^{0}$ and $g \in G \backslash\{1\}$ fixing $Z(x)$ pointwise, then necessarily $g$ is slack at $x$.

Proof. The result holds by Theorem 6.4, [5, Theorem 4.1], [17, Theorem 13.6] and [17, Theorem 14.10].

We are interested in determining the graded ideals of $A_{K}\left(\mathcal{G}_{(G, E)}\right)$ for a suitable grading. Notice that the function

$$
c: \mathcal{G}_{(G, E)} \rightarrow \mathbb{Z}
$$

such that $c([\alpha, g, \beta ; \beta \xi])=|\alpha|-|\beta|$ is a groupoid cocycle and hence determines a $\mathbb{Z}$-grading on $A_{K}\left(\mathcal{G}_{(G, E)}\right)$. However, in general $c^{-1}(0)$ will not be strongly effective so there might be $\mathbb{Z}$-graded ideals of $A_{K}\left(\mathcal{G}_{(G, E)}\right)$ that have trivial intersection with $A_{K}\left(\mathcal{G}_{(G, E)}^{(0)}\right)$. In what follows, we show that $A_{K}\left(\mathcal{G}_{(G, E)}\right)$ is also graded by the lag group.

\subsection{The lag function. Denote}

$$
G^{\infty}:=\prod_{n \in \mathbb{Z}^{+}} G \quad \text { and } \quad G^{(\infty)}:=\bigoplus_{n \in \mathbb{Z}^{+}} G .
$$

Denote the Corona group by

$$
\breve{G}:=G^{\infty} / G^{(\infty)} .
$$


Then the lag group of $\mathcal{G}_{(G, E)}$ is the semi-direct product $\breve{G} \rtimes_{\breve{\rho}} \mathbb{Z}$ where $\breve{\rho}$ is defined as follows: The right shift is the endomorphism $\rho: G^{\infty} \rightarrow G^{\infty}$ such that

$$
\rho(\mathbf{g})_{n}= \begin{cases}\mathbf{g}_{n-1} & \text { if } n>0 \\ 1 & \text { if } n=0 .\end{cases}
$$

Since classes in $G^{\infty}$ are invariant under $\rho$, we define $\breve{\rho}$ by passing to $\breve{G}$. Since $\breve{\rho}$ is an automorphism on $\breve{G}$ (with an abuse of notation) we have a homomorphism

$$
\breve{\rho}: \mathbb{Z} \rightarrow \text { Aut } \breve{G} \text { such that } \breve{\rho}(n)(\mathbf{g})=\breve{\rho}^{n}(\mathbf{g}) \text {. }
$$

Notation. We will write 1 for the identity in $G, \mathbf{1}$ for the identity in $\breve{G}$ and $\breve{\mathbf{1}}$ for the identity in $\breve{G} \rtimes_{\breve{\rho}} \mathbb{Z}$.

Now, we define a map $\Phi: G \times E^{\infty} \rightarrow G^{\infty}$ by the rule $\Phi(g, \xi)_{n}:=\varphi\left(g,\left.\xi\right|_{n-1}\right)[17$, Definition 8.9]. Thus,

Definition 6.6 ([17, Proposition 8.14]). The lag function is the map

$$
\begin{aligned}
& \ell: \mathcal{G}_{(G, E)} \rightarrow \quad \breve{G} \rtimes_{\breve{\rho}} \mathbb{Z} \\
& {[\alpha, g, \beta ; \beta \xi] \mapsto\left(\breve{\rho}^{|\alpha|}(\breve{\Phi}(g, \xi)),|\alpha|-|\beta|\right)}
\end{aligned}
$$

The lag function is a one-cocycle by [17, Proposition 8.14] and thus gives a grading on of our algebra.

6.5. Graded ideals. We would like to apply Theorem 5.3 to determine the $\breve{G} \rtimes_{\breve{\rho}} \mathbb{Z}$-graded ideals of $A_{K}\left(\mathcal{G}_{(G, E)}\right)$ for any field $K$. To this end, we need to verify that $\ell^{-1}(e)$ is strongly effective. In fact, we show it is principal.

Lemma 6.7. Suppose that $\mathcal{G}_{(G, E)}$ is Hausdorff. Let $e:=(\breve{\mathbf{1}}, 0)$ be the identity in $\breve{G} \rtimes_{\breve{\rho}} \mathbb{Z}$. Then $\ell^{-1}(e)=\left\{[\alpha, 1, \beta ; \beta \xi]: \alpha, \beta \in E^{*},|\alpha|=|\beta|\right\}$.

Proof. Let $\gamma \in \ell^{-1}(e)$. Then, $\gamma=[\alpha, g, \beta ; \beta \xi]$, and since $\ell(\gamma)=(\breve{\mathbf{1}}, 0)$, we have that $|\alpha|=|\beta|$. On the other side, $\breve{\mathbf{1}}=\breve{\rho}^{|\alpha|}(\breve{\Phi}(g, \xi))$ means that, up to a finite number of entries, the sequence $\left(g, \varphi\left(g,\left.\xi\right|_{1}\right), \varphi\left(g,\left.\xi\right|_{2}\right), \ldots, \varphi\left(g,\left.\xi\right|_{k}\right), \ldots\right)$ coincides with the sequence $(1,1, \ldots, 1, \ldots)$. This is equivalent to say that there exists $n \in \mathbb{N}$ such that $\varphi\left(g,\left.\xi\right|_{k}\right)=1$ for all $k \geq n$. Define $\tau:=\left.\xi\right|_{n}$. Then,

$$
[\alpha, g, \beta ; \beta \xi]=\left[\alpha \tau, \varphi(g, \tau), \beta \tau ; \beta \tau \xi_{[n+1, \infty)}\right]=\left[\alpha \tau, 1, \beta \tau ; \beta \tau \xi_{[n+1, \infty)}\right]
$$

as desired.

Corollary 6.8. Suppose that $\mathcal{G}_{(G, E)}$ is Hausdorff. Then, $\ell^{-1}(e)$ is principal.

Proof. Clearly, $\mathcal{G}_{(G, E)}^{(0)} \subseteq \ell^{-1}(e) \cap \operatorname{Iso}\left(\mathcal{G}_{(G, E)}\right)$. On the other side, if $\gamma \in \operatorname{Iso}\left(\mathcal{G}_{(G, E)}\right) \backslash \mathcal{G}_{(G, E)}^{(0)}$, then $\gamma=[\alpha, g, \alpha, \alpha \xi]$ with $g \cdot \xi=\xi$ but $\varphi\left(g,\left.\xi\right|_{n}\right) \neq 1$ for every $n \in \mathbb{N}$. By Lemma 6.7, $\gamma \notin \ell^{-1}(e)$, so we are done.

Thus we can apply Theorem 5.3 to see that the graded ideals in $A_{\mathbb{C}}\left(\mathcal{G}_{(G, E)}\right)$ are precisely the ideals of the form let $I(U):=A_{\mathbb{C}}\left(\mathcal{G}_{(G, E) \mid U}\right)$ for some open $\mathcal{S}_{G, E}$-invariant subset $U \subseteq$ $\mathcal{G}_{(G, E)}^{(0)}$.

Next we will show how graded ideals in $A_{\mathbb{C}}\left(\mathcal{G}_{(G, E)}\right)$ come from particular subsets of vertices of $E$. We say $H \subseteq E^{0}$ is hereditary if for any $w \in H$ such that there exists $e \in E^{1}$ 
with $r(e)=w$, then $s(e) \in H$ as well. We say $H$ is saturated if the following condition is satisfied:

$$
v \in E^{0} \text { such that } s\left(r^{-1}(v)\right) \subseteq H \Longrightarrow v \in H .
$$

Now suppose that $U$ is an open $\mathcal{S}_{G, E^{-}}$invariant subset of $\mathcal{G}_{G, E}^{(0)} \cong E^{\infty}$. Motiated by the graph algebra construction in [12, Theorem 3.3] (for example) define

$$
H_{U}:=\left\{x \in E^{0}: \Theta((x, 1, x), Z(x)) \subseteq U\right\} .
$$

Lemma 6.9. The set $H_{U}$ defined above is a G-invariant saturated hereditary set.

Proof. To see that $H_{U}$ is hereditary, fix $w \in H_{U}$ and $e \in E^{1}$ such that $r(e)=w$. It suffices to show that $\Theta(s(e), 1, s(e) ; Z(s(e))) \subseteq U$. Fix

$$
[s(e), 1, s(e) ; \zeta] \in \Theta(s(e), 1, s(e) ; Z(s(e))) .
$$

Notice

$$
s([e, 1, s(e) ; \zeta])=[s(e), 1, s(e) ; \zeta] \quad \text { and } \quad r([e, 1, s(e) ; \zeta])=[e, 1, e ; e \zeta] .
$$

Since $[e, 1, e ; e \zeta] \in \Theta(w, 1, w ; Z(w))$ we have $[e, 1, e ; e \zeta] \in U$ and hence $[s(e), 1, s(e) ; \zeta] \in U$ because $U$ is invariant.

To see that $H_{U}$ is saturated, fix $v$ such that $s\left(r^{-1}(v)\right) \subseteq H_{U}$. Notice that

$$
\Theta(v, 1, v ; Z(v))=\bigcup_{e \in r^{-1}(v)} \Theta(e, 1, e ; Z(e)) .
$$

We have $\Theta(s(e), 1, s(e) ; Z(s(e))) \subseteq U$. Using an argument similarly to the one in (6.10), we see that $\Theta(e, 1, e ; Z(e)) \subseteq U$. Thus $\Theta((v, 1, v), Z(v)) \subseteq U$ which means $v \in H_{U}$.

That $H_{U}$ is $G$-invariant follows from $U$ being $\mathcal{S}_{G, E}$-invariant.

Denote by $1_{x}$ the characteristic function $1_{\Theta((x, 1, x), Z(x))} \in A_{\mathbb{C}}\left(\mathcal{G}_{(G, E)}\right)$. Also, let $I\left(H_{U}\right)$ denote the ideal generated by $\left\{1_{x}\right\}_{x \in H_{U}}$.

Proposition 6.11. Suppose that $\mathcal{G}_{(G, E)}$ is Hausdorff. Let $U$ be an open $\mathcal{S}_{G, E}$-invariant subset in $E^{\infty}$. Then $I(U)=I\left(H_{U}\right)$.

Proof. To show the forward containment, it suffices to show $1_{\Theta((\alpha, 1, \alpha), Z(\alpha))}$ is in $I\left(H_{U}\right)$ for any $\Theta((\alpha, 1, \alpha), Z(\alpha)) \subseteq U$. First we claim that $\Theta((s(\alpha), 1, s(\alpha)), Z(s(\alpha))) \subseteq U$. To see this, fix

$$
[s(\alpha), 1, s(\alpha) ; \zeta] \in \Theta((s(\alpha), 1, s(\alpha)), Z(s(\alpha))
$$

Then we have

$$
s([\alpha, 1, s(\alpha) ; \zeta])=[s(\alpha), 1, s(\alpha) ; \zeta] \quad \text { and } \quad r([\alpha, 1, s(\alpha) ; \zeta])=[\alpha, 1, \alpha ; \alpha \zeta] \in U .
$$

Thus $[s(\alpha), 1, s(\alpha) ; \zeta] \in U$ by invariance, proving the claim. Therefore $s(\alpha) \in H_{U}$. Now

$$
1_{\Theta((\alpha, 1, \alpha), Z(\alpha))}=1_{\Theta((\alpha, 1, \alpha), Z(\alpha))} 1_{s(\alpha)}
$$

and hence $1_{\Theta((\alpha, 1, \alpha), Z(\alpha))} \in I\left(H_{U}\right)$.

For the reverse containment, notice that for any $x \in H_{U}$ we have $1_{x} \in I(U)$.

As an immediate consequence of Proposition 6.11, we conclude that

Corollary 6.12. If $U$ is an open $\mathcal{S}_{G, E}$-invariant subset of $E^{\infty}$, then $I(U)$ is a graded ideal of $A_{K}\left(\mathcal{G}_{(G, E)}\right)$ generated by a $G$-invariant saturated hereditary subset $H_{U}$ of $E^{0}$. 
Thus, the relevant information related to this concrete class of ideals relates to the ideal theory developed for graph $C^{*}$-algebras [20, Chapter 4] and Leavitt path algebras [1, Proposition $5.2 \&$ Theorem 5.3].

\section{EXAMPle: BoOlEAN DYNAMiCAL SYSTEMS}

$C^{*}$-algebras associated to Boolean dynamical systems were recently introduced by Carlsen, Ortega and the third author in [7]. Let us briefly recall the definition of these algebras.

7.1. The algebra. Let $\mathcal{B}$ be a Boolean algebra, we say that a map $\theta: \mathcal{B} \longrightarrow \mathcal{B}$ is an action on $\mathcal{B}$ if $\theta$ is a Boolean algebra homomorphism with $\theta(\emptyset)=\emptyset$. We say that the action has compact range if $\{\theta(A)\}_{A \in \mathcal{B}}$ has least upper-bound, that we will denote $\mathcal{R}_{\theta}$. Moreover, we say that the action has closed domain if there exists $\mathcal{D}_{\theta} \in \mathcal{B}$ such that $\theta\left(\mathcal{D}_{\theta}\right)=\mathcal{R}_{\theta}$

Given a set $\mathcal{L}$, and given any $n \in \mathbb{N}$, we define

$$
\left.\mathcal{L}^{n}=\left\{\left(\alpha_{1}, \ldots, \alpha_{n}\right): \alpha_{i} \in \mathcal{L}\right)\right\} \quad \text { and } \quad \mathcal{L}^{*}=\bigcup_{n=0}^{\infty} \mathcal{L}^{n},
$$

where $\mathcal{L}^{0}=\{\emptyset\}$. Given $\alpha \in \mathcal{L}^{n}$ for $n \geq 1$, we will write it as $\alpha=\alpha_{1} \cdots \alpha_{n}$ where $\alpha_{i} \in \mathcal{L}$.

A Boolean dynamical system on a Boolean algebra $\mathcal{B}$ is a triple $(\mathcal{B}, \mathcal{L}, \theta)$ such that $\mathcal{L}$ is a set, and $\left\{\theta_{\alpha}\right\}_{\alpha \in \mathcal{L}}$ is a set of actions on $\mathcal{B}$. Moreover, given $\alpha=\left(\alpha_{1}, \ldots, \alpha_{n}\right) \in \mathcal{L}^{\geq 1}$ the action $\theta_{\alpha}: \mathcal{B} \longrightarrow \mathcal{B}$ defined as $\theta_{\alpha}=\theta_{\alpha_{n}} \circ \cdots \circ \theta_{\alpha_{1}}$ has compact range and closed domain.

Given any $\alpha \in \mathcal{L}^{*}$, we will write $\mathcal{D}_{\alpha}:=\mathcal{D}_{\theta_{\alpha}}$ and $\mathcal{R}_{\alpha}:=\mathcal{R}_{\theta_{\alpha}}$. Also, when $\alpha=\emptyset$, we will define $\theta_{\emptyset}=\mathrm{Id}$, and we will formally assume that $\mathcal{R}_{\emptyset}=\mathcal{D}_{\emptyset}:=\bigcup_{A \in \mathcal{B}} A$, in order to guarantee that $A \subseteq \mathcal{R}_{\emptyset}$ for every $A \in \mathcal{B}$.

Let $(\mathcal{B}, \mathcal{L}, \theta)$ be a Boolean dynamical system. Given $B \in \mathcal{B}$ we define

$$
\Delta_{B}:=\left\{\alpha \in \mathcal{L}: \theta_{\alpha}(B) \neq \emptyset\right\} \quad \text { and } \quad \lambda_{B}:=\left|\Delta_{B}\right| .
$$

We say that $A \in \mathcal{B}$ is a regular set if given any $\emptyset \neq B \in \mathcal{B}$ with $B \subseteq A$ we have that $0<\lambda_{B}<\infty$, otherwise $A$ is called a singular set. We denote by $\mathcal{B}_{\text {reg }}$ the set of all regular sets where we will include $\emptyset$.

A Cuntz-Krieger representation of the Boolean dynamical system $(\mathcal{B}, \mathcal{L}, \theta)$ in a $C^{*}$ algebra $\mathcal{A}$ consists of a family of projections $\left\{P_{A}: A \in \mathcal{B}\right\}$ and partial isometries $\left\{S_{\alpha}\right.$ : $\alpha \in \mathcal{L}\}$ in $\mathcal{A}$, with the following properties:

(1) If $A, B \in \mathcal{B}$, then $P_{A} \cdot P_{B}=P_{A \cap B}$ and $P_{A \cup B}=P_{A}+P_{B}-P_{A \cap B}$, where $P_{\emptyset}=0$.

(2) If $\alpha \in \mathcal{L}$ and $A \in \mathcal{B}$, then $P_{A} \cdot S_{\alpha}=S_{\alpha} \cdot P_{\theta_{\alpha}(A)}$.

(3) If $\alpha, \beta \in \mathcal{L}$ then $S_{\alpha}^{*} \cdot S_{\beta}=\delta_{\alpha, \beta} \cdot P_{\mathcal{R}_{\alpha}}$.

(4) Given $A \in \mathcal{B}_{\text {reg }}$ we have that

$$
P_{A}=\sum_{\alpha \in \Delta_{A}} S_{\alpha} \cdot P_{\theta_{\alpha}(A)} \cdot S_{\alpha}^{*}
$$

A representation is called faithful if $P_{A} \neq 0$ for every $A \in \mathcal{B}$.

Given a representation $\left\{P_{A}, S_{\alpha}\right\}$ of a Boolean dynamical system $(\mathcal{B}, \mathcal{L}, \theta)$ in a $C^{*}$-algebra $\mathcal{A}$, we define $C^{*}\left(P_{A}, S_{\alpha}\right)$ to be the sub- $C^{*}$-algebra of $\mathcal{A}$ generated by $\left\{P_{A}, S_{\alpha}: A \in \mathcal{B}, \alpha \in\right.$ $\mathcal{L}\}$. 
A universal representation $\left\{p_{A}, s_{\alpha}\right\}$ of a Boolean dynamical system $(\mathcal{B}, \mathcal{L}, \theta)$ is a representation satisfying the following universal property: given a representation $\left\{P_{A}, S_{\alpha}\right\}$ of $(\mathcal{B}, \mathcal{L}, \theta)$ in a $C^{*}$-algebra $\mathcal{A}$, there exists a non-degenerate $*$-homomorphism $\pi_{S, P}$ : $C^{*}\left(p_{A}, s_{\alpha}\right) \longrightarrow \mathcal{A}$ such that $\pi_{S, P}\left(p_{A}\right)=P_{A}$ and $\pi_{S, P}\left(s_{\alpha}\right)=S_{\alpha}$ for $A \in \mathcal{B}$ and $\alpha \in \mathcal{L}$. We will set $C^{*}(\mathcal{B}, \mathcal{L}, \theta):=C^{*}\left(p_{A}, s_{\alpha}\right)$.

It is shown that

$$
T=T_{(\mathcal{B}, \mathcal{L}, \theta)}:=\left\{s_{\alpha} p_{A} s_{\beta}^{*}: \alpha, \beta \in \mathcal{L}^{*}, A \in \mathcal{B}, A \subseteq \mathcal{R}_{\alpha} \cap \mathcal{R}_{\beta} \neq \emptyset\right\} \cup\{0\} \subseteq C^{*}(\mathcal{B}, \mathcal{L}, \theta),
$$

is a $*$-inverse semigroup [7, Proposition 6.2] such that $C^{*}(\mathcal{B}, \mathcal{L}, \theta)$ is the closure of the linear span of $T$, and that it is $E^{*}$-unitary [7, Proposition 6.8]. Moreover, if $\mathcal{B}$ and $\mathcal{L}$ are countable sets, then $C^{*}(\mathcal{B}, \mathcal{L}, \theta)$ is $*$-isomorphic to $C^{*}\left(\mathcal{G}_{\text {tight }}(T)\right)[7$, Theorem 8.3], which is a Hausdorff [7, Lemma 8.2] amenable [7, Lemma 8.4] ample groupoid.

7.2. The Steinberg algebra. An argument analogous to that used for proving Theorem 6.4 shows

Theorem 7.1. The map

$$
\begin{aligned}
& \tau: \quad A_{R}\left(\mathcal{G}_{\text {tight }}(T)\right) \rightarrow R(\mathcal{B}, \mathcal{L}, \theta) \\
& 1_{\Theta\left(s_{\alpha}, \operatorname{Dom}\left(s_{\alpha}^{*} s_{\alpha}\right)\right)} \mapsto s_{\alpha} \\
& 1_{\Theta\left(p_{A}, \operatorname{Dom}\left(p_{A}\right)\right)} \mapsto p_{A}
\end{aligned}
$$

is an R-algebra isomorphism.

In particular, we can characterize simplicity of $A_{R}\left(\mathcal{G}_{\text {tight }}(T)\right)$. To do this, let us recall some definitions

Let $(\mathcal{B}, \mathcal{L}, \theta)$ be a Boolean dynamical system. Then:

- We say that the pair $(\alpha, A)$ with $\alpha=\alpha_{1} \cdots \alpha_{n} \in \mathcal{L}^{n}$ for some $n \geq 1$, and $\emptyset \neq A \in \mathcal{B}$ with $A \subseteq \mathcal{R}_{\alpha}$, is a cycle if given $k \in \mathbb{N} \cup\{0\}$ we have that $\theta_{\alpha^{k}}(A) \neq \emptyset$ and for every $\emptyset \neq B \subseteq \theta_{\alpha^{k}}(A)$ we have that $B \cap \theta_{\alpha}(B) \neq \emptyset$.

- A cycle $(\alpha, A)$ has no exits if given any $k \in \mathbb{N} \cup\{0\}$ we have that $\theta_{\alpha^{k} \alpha_{1} \cdots \alpha_{t}}(A) \in \mathcal{B}_{\text {reg }}$ with $\Delta_{\theta_{\alpha^{k} \alpha_{1} \cdots \alpha_{t}}(A)}=\left\{\alpha_{t+1}\right\}$ for $t<n$ and $\theta_{\alpha^{k+1}}(A) \in \mathcal{B}_{\text {reg }}$ with $\Delta_{\theta_{\alpha} k+1}(A)=\left\{\alpha_{1}\right\}$.

- We say that $(\mathcal{B}, \mathcal{L}, \theta)$ satisfies condition $\left(L_{\mathcal{B}}\right)$ if there is no cycle without exits.

- We say that an ideal $\mathcal{I}$ of $\mathcal{B}$ is hereditary if given $A \in \mathcal{I}$ and $\alpha \in \mathcal{L}$ then $\theta_{\alpha}(A) \in \mathcal{I}$.

- We also say that $\mathcal{I}$ is saturated if given $A \in \mathcal{B}_{\text {reg }}$ with $\theta_{\alpha}(A) \in \mathcal{I}$ for every $\alpha \in \Delta_{A}$ then $A \in \mathcal{I}$.

As a consequence, we have the following:

Corollary 7.2. If $(\mathcal{B}, \mathcal{L}, \theta)$ is a Boolean dynamical system such that $\mathcal{B}$ and $\mathcal{L}$ are countable, $K$ is a field and $K(\mathcal{B}, \mathcal{L}, \theta)$ is its associated $K$-algebra, then, the following statements are equivalent:

(1) $K(\mathcal{B}, \mathcal{L}, \theta)$ is simple.

(2) The following properties hold:

(a) $(\mathcal{B}, \mathcal{L}, \theta)$ satisfies condition $\left(L_{\mathcal{B}}\right)$, and

(b) The only hereditary and saturated ideals of $\mathcal{B}$ are $\emptyset$ and $\mathcal{B}$.

Proof. The result holds by Theorem 7.1, [5, Theorem 4.1], [7, Theorem 9.7] and [7, Theorem 9.15]. 
7.3. Graded ideals. For any field $K$, we can endow a structure of $\mathbb{Z}$-graded algebra on $A_{K}\left(\mathcal{G}_{\text {tight }}(T)\right)$ using the continuous cocycle

$$
c: \begin{array}{ccc}
\mathcal{G}_{\text {tight }}(T) & \rightarrow & \mathbb{Z} \\
{\left[s_{\alpha} p_{A} s_{\beta}^{*}, \xi\right]} & \mapsto|\alpha|-|\beta|
\end{array} .
$$

Hence, we apply Theorem 5.3 to determine the $\mathbb{Z}$-graded ideals of $A_{K}\left(\mathcal{G}_{\text {tight }}(T)\right)$ for any field $K$, provided we are able to prove that $c^{-1}(0)$ is strongly effective. We will prove that, in fact, $c^{-1}(0)$ is principal.

Lemma 7.3. The subgroupoid $c^{-1}(0)$ of $\mathcal{G}_{\text {tight }}(T)$ is principal.

Proof. First, we define the $*$-subsemigroup of $T$

$$
S=\left\{s_{\alpha} p_{A} s_{\beta}^{*} \in T:|\alpha|=|\beta|\right\} .
$$

Miming the arguments in [7, Section 7], one can show that

$$
\iota: S \rightarrow\left(C^{*}(\mathcal{B}, \mathcal{L}, \theta)^{\gamma}\right)^{1}
$$

is the universal tight representation of $S$ (where $\gamma: \mathbb{T} \rightarrow C^{*}(\mathcal{B}, \mathcal{L}, \theta)$ is the standard gauge action), and that $C^{*}(\mathcal{B}, \mathcal{L}, \theta)^{\gamma} \cong C^{*}\left(\mathcal{G}_{\text {tight }}(S)\right)$. Moreover, it is a simple exercise to prove that $\mathcal{G}_{\text {tight }}(S)$ and $c^{-1}(0)$ are topologically isomorphic.

Then, an adaptation of the proof of [19, Proposition 2.9] (see also the proof of [3, Theorem 4.4] or [4, Lemma 2.2]) shows that $C^{*}(\mathcal{B}, \mathcal{L}, \theta)^{\gamma}$ is a AF-algebra; we thank Eduard Ortega for turning our attention to this fact, and providing an accurate proof of it. Thus, [21, Proposition III.1.15 \& Remarks III.1.2] imply that $c^{-1}(0)$ is principal, as desired.

We then apply Theorem 5.3 to determine the $\mathbb{Z}$-graded ideals of $A_{K}\left(\mathcal{G}_{\text {tight }}(T)\right)$ for any field $K$. First, we recall some more definitions from [7]. Given a collection $\mathcal{I}$ of elements of $\mathcal{B}$ we define the hereditary expansion of $\mathcal{I}$ as

$$
\mathcal{H}(\mathcal{I}):=\left\{B \in \mathcal{B}: B \subseteq \bigcup_{i=1}^{n} \theta_{\alpha_{i}}\left(A_{i}\right) \text { where } A_{i} \in \mathcal{I} \text { and } \alpha_{i} \in \mathcal{L}^{*}\right\}
$$

Clearly, $\mathcal{H}(\mathcal{I})$ is the minimal hereditary ideal of $\mathcal{B}$ containing $\mathcal{I}$. Also, we define the saturation of $\mathcal{I}$, denoted by $\mathcal{S}(\mathcal{I})$, to be the minimal ideal of $\mathcal{B}$ generated by the set

$$
\bigcup_{n=0}^{\infty} \mathcal{S}^{[n]}(\mathcal{I})
$$

defined by recurrence on $n \in \mathbb{Z}^{+}$as follows:

(1) $\mathcal{S}^{[0]}(\mathcal{I}):=\mathcal{I}$

(2) For every $n \in \mathbb{N}, \mathcal{S}^{[n]}(\mathcal{I}):=\left\{B \in \mathcal{B}_{\text {reg }}: \theta_{\alpha}(B) \in \mathcal{S}^{[n-1]}(\mathcal{I})\right.$ for every $\left.\alpha \in \Delta_{B}\right\}$.

Observe that if $\mathcal{I}$ is hereditary, then $\mathcal{S}(\mathcal{I})$ is also hereditary. Therefore, given a collection $\mathcal{I}$ of elements of $\mathcal{B}, \mathcal{S}(\mathcal{H}(\mathcal{I}))$ is the minimal hereditary and saturated ideal of $\mathcal{B}$ containing $\mathcal{I}$.

Given any idempotent $e \in \mathcal{E}(T)$, let $D_{e}$ denote the domain of the (partial) action of $e$ on the space of tight filters of $\mathcal{E}(T)$, which is homeomorphic to $\mathcal{G}_{\text {tight }}(T)^{(0)}$. Also, we denote by $\mathcal{O}(e):=r\left(s^{-1}\left(D_{e}\right)\right)$ the orbit of $D_{e}$ by the action of $T$, which is an open invariant subset of $\mathcal{G}_{\text {tight }}(T)^{(0)}$. Since $\left\{D_{e}: e \in \mathcal{E}(T)\right\}$ is a basis of compact open sets of $\mathcal{G}_{\text {tight }}(T)^{(0)}$, then $\{\mathcal{O}(e): e \in \mathcal{E}(T)\}$ is a cover of the collection of open invariant sets of $\mathcal{G}_{\text {tight }}(T)^{(0)}$ such that any open invariant subset $U$ of $\mathcal{G}_{\text {tight }}(T)^{(0)}$ is a union of sets in this collection. 
Notice that, given any $e \in \mathcal{E}(T)$, $e$ is of the form $s_{\alpha} p_{A} s_{\alpha}^{*}$ for $\alpha \in \mathcal{L}^{*}$ and $A \subseteq \mathcal{R}_{\alpha}$. Thus, taking $s=s_{\alpha}^{*}$, we have that $s \cdot\left(D_{e}\right)=D_{\text {ses }}$, where $s e s^{*}=p_{A}$. Hence, for any $e \in \mathcal{E}(T)$ there exists a (unique) $A \in \mathcal{B}$ such that $\mathcal{O}(e)=\mathcal{O}\left(p_{A}\right)$.

In an analogous way to that of [7, Section 10], we introduce the following definitions.

Definition 7.4. Let $(\mathcal{B}, \mathcal{L}, \theta)$ be a (countable) Boolean dynamical system. Then:

(1) Given $U \subseteq \mathcal{G}_{\text {tight }}(T)^{(0)}$ an open invariant subset, we define

$$
\mathcal{H}_{U}:=\left\{A \in \mathcal{B}: D_{p_{A}} \in I(U)\right\}
$$

where $I(U)$ is the ideal of $A_{K}\left(\mathcal{G}_{\text {tight }}(T)\right)$ generated by $U$. Clearly, $\mathcal{H}_{U}$ is a hereditary and saturated subset of $\mathcal{B}$.

(2) Given any hereditary and saturated subset $\mathcal{H}$ of $\mathcal{B}$, we define

$$
U_{\mathcal{H}}:=\bigcup_{A \in \mathcal{H}} \mathcal{O}\left(p_{A}\right)
$$

which is clearly an open invariant subset of $\mathcal{G}_{\text {tight }}(T)^{(0)}$.

Proposition 7.5. Let $(\mathcal{B}, \mathcal{L}, \theta)$ be a (countable) Boolean dynamical system. Then:

(1) Given $U \subseteq \mathcal{G}_{\text {tight }}(T)^{(0)}$ an open invariant subset, $U=U_{\mathcal{H}_{U}}$.

(2) Given any hereditary and saturated subset $\mathcal{H}$ of $\mathcal{B}, \mathcal{H}=\mathcal{H}_{U_{\mathcal{H}}}$.

So, there is a lattice isomorphism between open invariant subsets of $\mathcal{G}_{\text {tight }}(T)^{(0)}$ and hereditary and saturated subset of $(\mathcal{B}, \mathcal{L}, \theta)$.

Proof. (1) Take $U \subseteq \mathcal{G}_{\text {tight }}(T)^{(0)}$ an open invariant subset. Since $A \in \mathcal{H}_{U}$ if and only if $D_{p_{A}} \subseteq U$ and $U$ is invariant, we have that $\mathcal{O}\left(p_{A}\right) \subseteq U$. Thus, as $U_{\mathcal{H}_{U}}=\bigcup_{A \in \mathcal{H}_{U}} \mathcal{O}\left(p_{A}\right)$, we have that $U_{\mathcal{H}_{U}} \subseteq U$. On the other side, if $e \in T$ is an idempotent and $D_{e} \subseteq U$, then there exists a unique $A \in \mathcal{B}$ such that $\mathcal{O}(e)=\mathcal{O}\left(p_{A}\right)$, whence $D_{p_{A}} \subseteq U$. Thus, $D_{e} \subseteq \mathcal{O}(e)=\mathcal{O}\left(p_{A}\right) \subseteq U_{\mathcal{H}_{U}}$. Hence, $U \subseteq U_{\mathcal{H}_{U}}$, so we are done.

(2) Given any hereditary and saturated subset $\mathcal{H}$, we have that

$$
\mathcal{H}_{U_{\mathcal{H}}}=\left\{A \in \mathcal{B}: D_{p_{A}} \subseteq \bigcup_{B \in \mathcal{H}} \mathcal{O}\left(p_{B}\right)\right\}
$$

Thus, $\mathcal{H} \subseteq \mathcal{H}_{U_{\mathcal{H}}}$ trivially. On the other side, if $A \in \mathcal{H}_{U_{\mathcal{H}}}$, then $D_{p_{A}} \subseteq \bigcup_{B \in \mathcal{H}} \mathcal{O}\left(p_{B}\right)$. Since $D_{p_{A}}$ is compact, there exists $B_{1}, \ldots, B_{n} \in \mathcal{H}$ such that $D_{p_{A}} \subseteq \bigcup_{i=1}^{n} \mathcal{O}\left(p_{B_{i}}\right)$. Since $\mathcal{O}\left(p_{B}\right)=\bigcup_{s \in T} D_{s p_{B} s^{*}}$, again by compactness, for each $1 \leq i \leq n$ there exists $s_{i 1}, \ldots, s_{i m_{i}} \in$ $T$ such that $D_{p_{A}} \subseteq \bigcup_{i=1}^{n} \bigcup_{j=1}^{m_{i}} D_{s_{i j} p_{B_{i}} s_{i j}^{*}}$. Since the elements of $T$ are of the form $s_{\alpha} p_{A} s_{\beta}^{*}$ with $\alpha, \beta \in \mathcal{L}^{*}$ and $B \subseteq \mathcal{R}_{\alpha} \cap \mathcal{R}_{\beta}$, we can assume, relabelling if necessary, that there exist $\bigcup_{i=1}^{n}\left\{\alpha_{i 1}, \ldots, \alpha_{i m_{i}}\right\} \subseteq \mathcal{L}^{*}$ such that

$$
D_{p_{A}} \subseteq \bigcup_{i=1}^{n} \bigcup_{j=1}^{m_{i}} D_{s_{\alpha_{i j}} p_{\theta_{\alpha_{i j}}\left(B_{i}\right)} s_{\alpha_{i j}}^{*}}
$$


By [16, Proposition 3.7], this is equivalent to say that $\bigcup_{i=1}^{n} \bigcup_{j=1}^{m_{i}}\left\{s_{\alpha_{i j}} p_{\theta_{\alpha_{i j}}\left(B_{i}\right)} s_{\alpha_{i j}}^{*}\right\}$ is an outer cover of $p_{A}$. Then, applying the same argument as in $[7$, Theorem 9.5, proof of $(3) \Rightarrow(1)]$, we conclude that there exists $N \in \mathbb{N}$ such that $A \in \mathcal{S}^{[N]}\left(\mathcal{H}\left(B_{1}, \ldots, B_{N}\right)\right) \subseteq \mathcal{H}$. Hence, $\mathcal{H}_{U_{\mathcal{H}}} \subseteq \mathcal{H}$, so we are done.

As a consequence of Proposition 7.5 and Theorem 5.3, we conclude

Theorem 7.6. Given a (countable) Boolean dynamical system $(\mathcal{B}, \mathcal{L}, \theta)$ and a field $K$, the lattice of $\mathbb{Z}$-graded ideals of $A_{K}\left(\mathcal{G}_{\text {tight }}(T)\right)$ is isomorphic to the lattice of hereditary and saturated subset of $C^{*}(\mathcal{B}, \mathcal{L}, \theta)$.

Observe that Theorem 7.6 is analog to [7, Theorem 10.12], where the result is proved for $C^{*}$-algebras of Boolean dynamical systems.

\section{REFERENCES}

[1] P. Ara, M.A. Moreno, E. Pardo, Nonstable K-Theory for graph algebras, Algebras Represent. Theory. 10 (2007), 157-178.

[2] G. Aranda-Pino, E. Pardo, M. Siles-Molina, Exchange Leavitt path algebras and stable rank, J. Algebra 305 (2006), 912-936.

[3] T. Bates, D. PAsk, $C^{*}$-algebras of labelled graphs. II. Simplicity results, Math. Scand. 104 (2009), no. 2, 249-274.

[4] T. Bates, D. Pask, I. Raeburn, W. Szymański, The $C^{*}$-algebras of row-finite graphs, New York J. Math. 6 (2000), 307-324.

[5] J. Brown, L.O. Clark, C. Farthing, A. Sims, Simplicity of algebras associated to étale groupoids, Semigroup Forum 88 (2014), 433-452.

[6] J.H. Brown, G. Nagy, S. Reznikoff, A. Sims and D.P. Williams, Cartan subalgebras in $C^{*}$-algebras of Hausdorff étale groupoids, Integral Equations Operator Theory 85 (2016), 109-126.

[7] T.M. Carlsen, E. Ortega and E. Pardo, $C^{*}$-algebras associated to Boolean dynamical systems, arXiv:1510.06718v2.

[8] L.O. Clark and C. Edie-Michell, Uniqueness theorems for Steinberg algebras, Algebr. Represnt. Theor. 18 (2015) 907-916.

[9] L.O. Clark, C. Edie-Michell, A. an Huef and A. Sims, Ideals of Steinberg algebras of strongly effective groupoids, with applications to Leavitt path algebras, arXiv:1601.07238.

[10] L.O. Clark, C. Farthing, A. Sims and M. Tomforde, A groupoid generalization of Leavitt path algebras, Semigroup Forum, 89 (2014), 501-517.

[11] L.O. Clark, C. Gil Canto and A. Nasr-Isfahani, The cycline subalgebra of a Kumjian-Pask algebra, arXiv:1603.00508.

[12] L.O. Clark, D. Martín Barquero, C. Martín González and M. Siles Molina, Using Steinberg algebras to study decomposability of Leavitt path algebras, arXiv;1603:1033v1.

[13] R.Exel, Non-Hausdorff étale groupoids, Proc. Amer. Math. Soc. 139 (2011), 897-907.

[14] R. Exel, Inverse semigroups and combinatorial $C^{*}$-algebras, Bull. Braz. Math. Soc. 39 (2008), 191-313.

[15] R. ExeL, Reconstructing a totally disconnected groupoid from its ample semigroup, Proc. Amer. Math. Soc. 138 (2008), 2991-3001.

[16] R. Exel, E. PARdo, The tight groupoid of an inverse semigroup, Semigroup Forum 92 (2016), 274-303.

[17] R. Exel And E. PARdo, Self-similar graphs, a unified treatment of Katsura and Nekrashevych $C^{*}$-algebras, arXiv:1409.1107v2.

[18] C. Gil Canto, A. NAsR-Isfahani, The maximal commutative subalgebra of a Leavitt path algebra, arXiv: 1510.03992 .

[19] E. Ortega, Simple Cuntz-Krieger Boolean algebras, Draft (2016).

[20] I. Raeburn, "Graph Algebras", CBMS Reg. Conf. Ser. Math., vol. 103, Amer. Math. Soc., Providence, RI, 2005. 
[21] J. Renault, "A groupoid approach to $C^{*}$-algebras", Lecture Notes in Mathematics 793, Springer, Berlin, 1980.

[22] B. Steinberg, A groupoid approach to discrete inverse semigroup algebras, Adv. Math. 223 (2010) 689-727.

[23] B. Steinberg, Simplicity, primitivity and semiprimitivity of étale groupoid algebras with applications to inverse semigroup algebras, J. Pure Appl. Algebra 220 (2016), 1035-1054.

Department of Mathematics and Statistics, University of Otago, PO Box 56, Dunedin 9054, NeW ZEALAND

E-mail address: lclark@maths.otago.ac.nz

Departamento de Matemática, Universidade Federal de Santa Catarina, 88040-970 FloRIANÓPOLIS SC, BRAZIL

E-mail address: exel@mtm.ufsc.br

URL: http://www.mtm.ufsc.br/ exel/

Departamento de Matemáticas, Facultad de Ciencias, Universidad de Cádiz, Campus de Puerto Real, 11510 Puerto Real (CÁdiz), Spain.

E-mail address: enrique.pardo@uca.es

$U R L:$ https://sites.google.com/a/gm.uca.es/enrique-pardo-s-home-page/ 